\title{
Non-rigid Image Registration with Uniform Gradient Spherical Patterns
}

\author{
Shu Liao and Albert C.S. Chung \\ Lo Kwee-Seong Medical Image Analysis Laboratory, \\ Department of Computer Science and Engineering, \\ The Hong Kong University of Science and Technology, Hong Kong \\ liaoshu@cse.ust.hk, achung@cse.ust.hk
}

\begin{abstract}
In this paper, we propose a new feature based non-rigid image registration method for dealing with two important issues. First, in order to establish reliable anatomical correspondence between template and subject images, efficient and distinctive region descriptor is needed as intensity information alone maybe insufficient. Second, since interference factors such as monotonic gray-level bias fields are commonly existed during the imaging process, the registration algorithm should be robust against such factors. There are two main contributions presented in this paper. (1) A new region descriptor, named uniform gradient spherical pattern (UGSP), is proposed to extract the geometric features from input images. UGSP encodes second order voxel interaction information. (2) The UGSP feature is rotation and monotonic gray-level bias field invariant. The proposed method is integrated with the Markov random field (MRF) labeling framework to formulate the registration process. The $\alpha$ expansion algorithm is used to optimize the corresponding MRF energy function. The proposed method is evaluated on both the simulated and real 3D databases obtained from BrainWeb and IBSR respectively and compared with other state-of-the-art registration methods. Experimental results show that the proposed method gives the highest registration accuracy among all the compared methods on both databases.
\end{abstract}

\section{Introduction}

Non-rigid image registration is an active research topic during the last decade. It plays an important role in group analysis, image-guided surgery, atlas superposition and etc. During the last decade, many novel methods were proposed to tackle the non-rigid image registration problem. They can be generally classified into three categories: (1) Landmark based methods; (2) Intensity based methods and (3) Feature based methods. Landmark based registration methods [12] first select a set of landmark points from template and subject images. Then the optimum transformation is estimated based on the features extracted from those landmark points. In order to produce accurate registration results, a large number of landmark points are required, which brings additional computation burdens. Intensity based registration methods are usually fully automatic. They define similarity measure metrics based on the intensity distributions of input images to guide 
registration 34 . However, intensity similarity does not necessarily equivalent to anatomical similarity. Feature based registration methods use feature vectors to represent each voxel and the registration process is modeled as a feature matching and optimization problem. HAMMER [5] is a representative of this class of methods. The registration accuracy heavily depends on the feature vectors used.

Though the non-rigid image registration problem has been intensively studied during the last decade, it still remains as a challenging task. More precisely, two important issues arise in recent years: First, using intensity information only to characterize anatomical properties of brain images may be insufficient as analyzed in 6]. Therefore, new anatomical descriptor is needed to capture the geometric properties of different anatomical structures. Second, the registration approach should be robust against monotonic gray-level bias fields. Otherwise the algorithm may prefer to align the bias fields between two images instead of aligning their anatomical structures as stated in [7].

To this end, a new feature based non-rigid image registration method is proposed in this paper to deal with these two issues. The major contributions of this paper lie in the following aspects. First, a new region descriptor, named uniform gradient spherical pattern (UGSP), is designed as signatures for each voxel. UGSP encodes second order voxel interaction information. Second, UGSP is rotation and monotonic gray-level bias fields invariant. The UGSP feature is integrated with the Markov random field (MRF) labeling framework to formulate the registration process in this paper. The $\alpha$-expansion algorithm is used to optimize the corresponding energy function. The proposed method is evaluated on both the simulated and read 3D datasets obtained from BrainWeb and IBSR respectively. Experimental results show that the proposed method achieves the highest registration accuracy among all the compared methods.

\section{Uniform Gradient Spherical Patterns}

In this section, we describe the new region descriptor, called uniform gradient spherical patterns (UGSP), which is derived based on the local binary patterns (LBP) 8], analyze its properties and show how to use it to represent each voxel as signatures.

Suppose for an input image $G$. For each voxel $v_{c} \in G$ a spherical neighborhood is defined centered at $v_{c}$ with radius $R$. Also, $N$ samples are taken on the surface of the sphere by using the sampling method proposed in [9], denote them as $v_{i}$ $(\mathrm{i}=1,2, \ldots, \mathrm{N})$. For $v_{i}(\mathrm{i}=1,2, \ldots, \mathrm{N})$ which does not exactly fall in the $3 \mathrm{D}$ image grid its intensity is interpolated by tri-linear interpolation. Let $\nabla v_{i}$ denote the gradient of $v_{i}$. Then a rotation invariant gradient orientation measure of each neighboring voxel $v_{i}$ is defined by Equation 1, which is the angle between $\nabla v_{i}$ and $v_{c}-v_{i}$, denote as $\theta_{v_{i}}$.

$$
\theta_{v_{i}}=\arccos \frac{\nabla v_{i} \cdot\left(v_{c}-v_{i}\right)}{\left|\nabla v_{i}\right| \cdot\left|v_{c}-v_{i}\right|},
$$

where $\left|\nabla v_{i}\right|$ and $\left|v_{c}-v_{i}\right|$ denote the magnitudes of $\nabla v_{i}$ and $v_{c}-v_{i}$ respectively. 


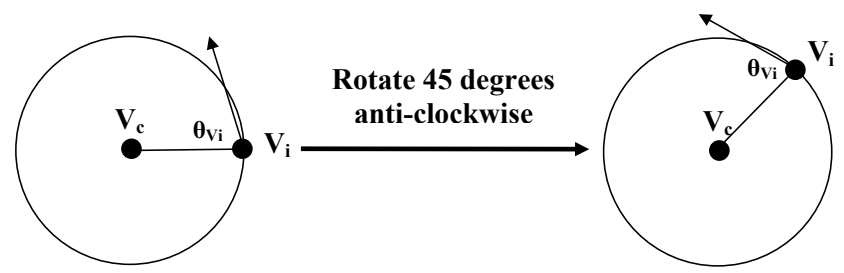

Fig. 1. A $2 \mathrm{D}$ example demonstrating the rotation invariant property of $\theta_{v_{i}} \cdot v_{c}$ is the center voxel, $v_{i}$ is a neighboring voxel. The black arrow pointing out from $v_{i}$ denotes the gradient vector of $v_{i}$. It is shown that the $\theta_{v_{i}}$ remains the same before and after rotating 45 degrees anti-clockwise.

$\theta_{v_{i}}$ is rotation invariant as it is the angle of the gradient of $v_{i}$ relative to the direction of $v_{c}-v_{i}$. Figure 1 shows a $2 \mathrm{D}$ example of $\theta_{v_{i}}$ before and after rotation of 45 degrees. It is shown that $\theta_{v_{i}}$ remains the same.

Then, the space of $\theta_{v_{i}}$ is uniformly partitioned into four subspaces. Each voxel is assigned with a label based on which subspace of $\theta_{v_{i}}$ it belongs. That is, each neighboring voxel is assigned with a label according to Equation 2.

$$
l\left(v_{i}\right)=\left\{\begin{array}{ccc}
1, & \text { if } \quad \theta_{v_{i}} \in\left[0, \frac{\pi}{4}\right), \\
2, & \text { if } \quad \theta_{v_{i}} \in\left[\frac{\pi}{4}, \frac{\pi}{2}\right), \\
3, & \text { if } \quad \theta_{v_{i}} \in\left[\frac{\pi}{2}, \frac{3 \pi}{4}\right), \\
4, & \text { if } \quad \theta_{v_{i}} \in\left[\frac{3 \pi}{4}, \pi\right] .
\end{array}\right.
$$

Then the basic gradient spherical pattern (BGSP) is defined based on Equations 1 and 2 ,

Definition 1. Basic gradient spherical pattern (BGSP) is the labeled spherical surface obtained from the original spherical neighborhood centered at the reference voxel by using Equations 1 and [2.

Besides rotation invariance, BGSP is also monotonic gray-level transformation invariant. As monotonic gray-level transformations affect the gradient magnitude of each voxel, the orientation of the gradient remains the same. Therefore, the angle $\theta_{v_{i}}$ between the directions of the gradient vector of $v_{i}$ and the vector $v_{c}-v_{i}$ also remains the same.

However, some of the BGSPs' occurrences are too sparse to reliably reflect the geometric features of input images. Therefore, a subset of BGSPs which represent the fundamental image structures, named uniform gradient spherical patterns (UGSP), are used as signatures of each voxel. Before we define UGSP, we first give the definition of "uniform region" as follows:

Definition 2. Uniform region is the area on the surface of BGSP where all the voxels belong to this area have the same label defined in Equation 2 .

Then, UGSP is defined as follows: 


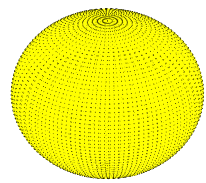

(a)

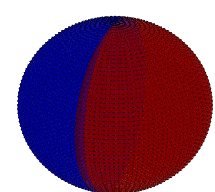

(b)

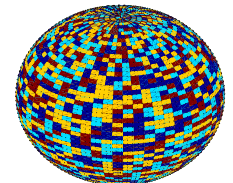

(c)

Fig. 2. (a) An example of UGSP, its surface can be covered by only one uniform region; (b) An example of UGSP, its surface can be covered by two uniform regions; (c) An example of non-UGSP, its surface cannot be covered by less than or equal to two uniform regions. Different colors represent different labels of each voxel.

\section{$\overline{\text { Algorithm 1. Determining whether a BGSP is a UGSP }}$}

Input: A BGSP with radius $R$ and $N$ neighboring voxels on the surface.

Output: true or false (whether the input is a UGSP or not).

1. Initialize all the neighboring voxels as unflagged.

2. $\quad$ FOR $\mathrm{j}=1$ to 2

3. IF all the neighboring voxels are flagged

4. Break;

5. ElSE

6. Randomly select an unflagged neighboring voxel $v_{i}$ on the surface of the input BGSP.

7. $\quad$ Find the largest connected component $C_{j}$ starting from $v_{i}$ based on its label $l\left(v_{i}\right)$ using the Breadth First Search (BFS), set a flag for each voxel belonging to that largest connected component. Two neighboring voxels $v_{m}$ and $v_{n}$ are

8. $\quad$ END IF considered to be connected if and only if $l\left(v_{m}\right)==l\left(v_{n}\right)$.

9. END FOR

10. IF all the neighboring voxels are flagged

11. Return true

12. EISE

13. Return false

14. END IF

Definition 3. Uniform gradient spherical patterns (UGSPs) are BGSPs whose surfaces can be covered by AT MOST two uniform regions.

For instance, Figure 2(a) is a UGSP as its surface can be covered by only one uniform region. Figure 2(b) is also a UGSP as its surface can be covered by two uniform regions. Figure 2(c) is not a UGSP as its surface cannot be covered by less than or equal to two uniform regions.

UGSP has physical meaning to mirror fundamental image structures. For example, Figure 2(a) denotes that there is an edge at a specific direction as all the neighboring voxels' gradient orientation measures are the same. Figure 2(b) represents that there is a corner formed by two edges. In this paper, all the non-uniform gradient spherical patterns are treated as a single type of pattern. Algorithm 1 is the procedure to determine whether a BGSP is a UGSP or not.

UGSP encodes second order information. The gradient orientation measure defined in Equation 1 already contains the first order interaction information 


\begin{abstract}
Algorithm 2. Calculate the UGSP Feature for each Voxel
Input: An input image $G$, a local cubic square window $W$ for each voxel, the UGSP radius $R$ and the number of neighboring samples $N$.
\end{abstract}

Output: A vector image $K$, each voxel is represented by a UGSP signature.

1. FOR each voxel $v \in G$

2. $\quad$ SubVolume $=(W$ center at $v)$

3. Initialize a new feature histogram, $H\left[0 \ldots\left(N-\left\lceil\frac{N}{2}\right\rceil+1\right)\right]=0$

4. FOR each voxel $t \in$ SubVolume

5. Calculate its corresponding BGSP $Q_{t}$ with parameters $R$ and $N$

6. Determine whether $Q_{t}$ is a UGSP or not using Algorithm 1

7. IF $Q_{t}$ is a UGSP

8. $\quad S=$ Size of the largest connected component in $Q_{t}$

9. $\quad$ PatternID $=S-\left\lceil\frac{N}{2}\right\rceil$

10. $\quad H[$ PatternID $]=H[$ Pattern $I D]+1$

11. ELSE

12. $H\left[N-\left\lceil\frac{N}{2}\right\rceil+1\right]=H\left[N-\left\lceil\frac{N}{2}\right\rceil+1\right]+1$

13. END IF

14. END FOR

15. Normalize $H\left[0 \ldots\left(N-\left\lceil\frac{N}{2}\right\rceil+1\right)\right]$ such that $\sum_{i=0}^{N-\left\lceil\frac{N}{2}\right\rceil+1} H[i]=1$

16. $\quad K(v)=H\left[0 \ldots\left(N-\left\lceil\frac{N}{2}\right\rceil+1\right)\right]$

17. END FOR

18. Return $K$

between voxels. During the operation of finding the connected components in Algorithm 1, higher order voxel information is considered based on the first order information embedded in the label of each voxel. While LBP [8] only considers the first order information as it only compares the intensity differences between the neighboring voxels and the center voxel. Therefore, UGSP has higher order information layer than LBP.

In this paper, the type ID of a UGSP is determined based on the size of the largest connected component of the UGSP (e.g. $\max \left(\left|C_{1}\right|,\left|C_{2}\right|\right), C_{1}$ and $C_{2}$ are calculated in operation 7 in Algorithm 1, $\left|C_{1}\right|$ and $\left|C_{2}\right|$ respectively denote the sizes of $C_{1}$ and $C_{2}$ ). The procedure for calculating the UGSP feature signatures for each voxel of the input image is presented in Algorithm 2.

A more detail UGSP type classification can be achieved if we also consider the label type and shape of the largest connected component. However, it will make the UGSP feature histogram too sparse as there are too many possible combinations and some of the pattern's occurrence frequency are too small. Radius $R$ in the Algorithm 2 affects the scale of interest. In this paper, the radius $R$ is set by the best scale selection principle in [10]. 


\section{MRF Modeling for Registration}

In recent years, it is shown that the registration process can be robustly formulated as a MRF labeling problem 11. In this paper, the UGSP feature is integrated with the MRF framework to drive the registration.

The general form of the MRF energy function can be defined by Equation 3 .

$$
\begin{aligned}
E_{f} & =E_{\text {data }}+E_{\text {smoothness }} \\
& =\sum_{p \in \Omega} D_{p}\left(l_{p}\right)+\sum_{(p, q) \in N} V_{p, q}\left(l_{p}, l_{q}\right),
\end{aligned}
$$

where $\Omega$ is the set of voxels, $N$ is the neighborhood system defined in $\Omega$. In this paper, the 4 -connected neighborhood system is used. $D_{p}\left(l_{p}\right)$ is the energy function associated with the data term. It penalizes the cost of assigning label $l_{p}$ to voxel $p . V_{p, q}\left(l_{p}, l_{q}\right)$ is the energy function associated with the smoothness term, and it penalizes the cost of label discrepancy between two neighboring voxels.

The registration problem is transformed to the MRF labeling problem by quantizing the deformation space. A discrete set of labels $L \in\left\{l^{1}, l^{2}, \ldots, l^{n}\right\}$ is defined. Each label $l^{i}(1 \leq i \leq n)$ corresponds to a displacement vector $\boldsymbol{d}_{\boldsymbol{i}}$. Assigning the voxel $p$ with label $l_{p}$ means moving $p$ to a new position by the displacement vector $\boldsymbol{d}_{\boldsymbol{l}_{p}}$. We follow the quantization step in [12, where each voxel can be moved from the original position bounded by a discretized window $\Psi=\{0, \pm s, \pm 2 s, \ldots, \pm w s\}^{d}$ of dimension $d$.

The energy function $D_{p}\left(l_{p}\right)$ associated with the data term is defined based on the UGSP features as:

$$
\begin{aligned}
D_{p}\left(l_{p}\right) & =D_{p}\left(G_{\text {template }}(\boldsymbol{p}), G_{\text {subject }}\left(\boldsymbol{p}+\boldsymbol{d}_{\boldsymbol{l}_{\boldsymbol{p}}}\right)\right. \\
& =D_{p}\left(K_{\text {template }}(\boldsymbol{p}), K_{\text {subject }}\left(\boldsymbol{p}+\boldsymbol{d}_{\boldsymbol{l}_{\boldsymbol{p}}}\right)\right) \\
& =J S D\left(K_{\text {template }}(\boldsymbol{p}) \| K_{\text {subject }}\left(\boldsymbol{p}+\boldsymbol{d}_{\boldsymbol{l}_{\boldsymbol{p}}}\right)\right),
\end{aligned}
$$

where $G_{\text {template }}$ is the template image, $G_{\text {subject }}$ is the subject image, $K_{\text {template }}$ and $K_{\text {subject }}$ are the UGSP feature images of $G_{\text {template }}$ and $G_{\text {subject }}$ respectively at the current iteration calculated by Algorithm 2, $J S D(\cdot)$ is the Jensen-Shannon divergence measure.

The smoothness energy function $V_{p, q}\left(l_{p}, l_{q}\right)$ is defined as:

$$
V_{p, q}\left(l_{p}, l_{q}\right)=\min \left(\lambda,\left|\boldsymbol{d}_{\boldsymbol{l}_{p}}-\boldsymbol{d}_{\boldsymbol{l}_{q}}\right|\right),
$$

where $\lambda$ is a constant represents the maximum penalty. It is the piece-wise truncated absolute distance.

The alpha-expansion algorithm 13 is applied to minimize the energy function defined in Equation 3 with the data term energy function and smoothness term energy function defined in Equations 4 and 5 . 


\section{Experimental Results}

The proposed method is evaluated on both the simulated and real 3D databases obtained from BrainWeb 1 and IBSR 2 respectively. In all experiments, the subvolume window sizes $W$ was set to $16 \times 16 \times 16$ in Algorithm 2 the number of neighboring samples $N$ for each UGSP was 60 . The 3D displacement window used in this paper was $\Psi=\{0, \pm 1, \pm 2, \ldots, \pm 12\}^{3}$. The maximum penalty parameter $\lambda$ defined in Equation 5 was set to 20. The proposed method is also compared with three widely used approaches: FFD [3], Demons [4] and HAMMER [5. In all the experiments, the control point spacing of FFD was set to $2.5 \mathrm{~mm}$. For Demons, the displacement field was smoothed by a unit variance Gaussian kernel. The elementary demon forces were computed by the optical flow equation [4.

\subsection{Experiment on Simulated Data}

Twenty image volumes from different subjects were obtained from BrainWeb. One of the image volumes was selected as the template image, and the others were used as the subject images. The resolution of each image was $256 \times 256 \times 181$ voxels. The segmentation results of each image for three classes of tissues: white matter (WM), gray matter (GM) and the cerebrospinal fluid (CSF) are also provided by the BrainWeb. The skull of each brain image was removed before registration by using the software Brain Suite version 2 obtained from USC 3 as it is a required step for HAMMER 5 to be compared in this paper.

The tissue overlap of GM, WM and CSF between the template and transformed subject images [14 was adopted as the evaluation function. The evaluation function is defined as $P=\frac{N(A \cap B)}{N(A \cup B)}$, where $A$ and $B$ denote the regions of a specific tissue in two images. The average values and standard deviations of $P$ for GM, WM and CSF before registration, registration after using FFD [3], Demons [4, HAMMER [5] and the proposed method are listed in Table 1.

It is observed in Table 1 that the proposed method has the highest value of $P$ among all the methods in this comparison. It reflects that the proposed method can give high registration accuracy.

\subsection{Experiment on Real Data}

The proposed method was also evaluated on the real datasets obtained from IBSR. Twenty skull-stripped image volumes with segmentation results were obtained from IBSR. The size of each volume was around $256 \times 256 \times 64$ voxels. The experimental settings were similar to the settings described in Section 4.1 Again, the tissue overlap evaluation function was used to measure the registration accuracy of different approaches. Table 2 lists the tissue overlap function value of $P$ for various methods.

\footnotetext{
${ }^{1}$ http://www.bic.mni.mcgill.ca/brainweb/

${ }^{2}$ http://www.cma.mgh.harvard.edu/ibsr/

${ }^{3}$ http://brainsuite.usc.edu/
} 
Table 1. The mean values of $P$ and SDs of GM, WM and CSF with different methods on the simulated $3 \mathrm{D}$ database. $B R$ denotes before registration.

\begin{tabular}{|c|c|c|c|c|c|}
\hline Tissue & BR & FFD & Demons & HAMMER & UGSP \\
\hline Gray & $0.41923 \pm 0.07$ & $0.75284 \pm 0.05$ & $0.78522 \pm 0.04$ & $0.80273 \pm 0.05$ & $0.84452 \pm 0.04$ \\
White & $0.48344 \pm 0.03$ & $0.76409 \pm 0.06$ & $0.78376 \pm 0.05$ & $0.81296 \pm 0.04$ & $0.83201 \pm 0.07$ \\
CSF & $0.37025 \pm 0.06$ & $0.72936 \pm 0.06$ & $0.75784 \pm 0.04$ & $0.76553 \pm 0.05$ & $0.80146 \pm 0.05$ \\
\hline
\end{tabular}

Table 2. The mean values of $P$ and SDs of GM, WM and CSF with different methods on the real $3 \mathrm{D}$ database. $B R$ denotes before registration.

\begin{tabular}{|c|c|c|c|c|c|}
\hline Tissue & BR & FFD & Demons & HAMMER & UGSP \\
\hline Gray & $0.54082 \pm 0.06$ & $0.75193 \pm 0.05$ & $0.77631 \pm 0.05$ & $0.79063 \pm 0.06$ & $0.83167 \pm 0.05$ \\
White & $0.52147 \pm 0.05$ & $0.76728 \pm 0.07$ & $0.77382 \pm 0.06$ & $0.80274 \pm 0.04$ & $0.83639 \pm 0.04$ \\
CSF & $0.33094 \pm 0.07$ & $0.73425 \pm 0.05$ & $0.76813 \pm 0.05$ & $0.77153 \pm 0.05$ & $0.80125 \pm 0.05$ \\
\hline
\end{tabular}

It is shown that the proposed method still has the largest value of $P$ among all the methods in the comparison and it strongly implies the registration accuracy of the proposed method.

\section{Conclusion}

In this paper, a new feature based non-rigid image registration method is proposed. The proposed method is based on a new type of image feature, named uniform gradient spherical patterns (UGSP). UGSP encodes second order information to capture the geometric properties around each voxel. UGSP is rotation invariant and monotonic gray-level transformation invariant. The UGSP feature is integrated with the Markov random field (MRF) labeling framework to formulate the registration process. The proposed method is evaluated on both the simulated and real 3D datasets obtained from BrainWeb and IBSR respectively. It is also compared with other state-of-the-art registration methods. Experimental results show that the proposed method has the highest registration accuracies among all the compared methods on both the simulated and real 3D datasets.

\section{References}

1. Rohr, K.: Image registration based on thin-plate splines and local estimates of anisotropic landmark localization uncertainties. In: Wells, W.M., Colchester, A.C.F., Delp, S.L. (eds.) MICCAI 1998. LNCS, vol. 1496, pp. 1174-1183. Springer, Heidelberg (1998)

2. Thompson, P., Toga, A.W.: A surface-based technique for warping threedimensional images of the brain. TMI 15, 402-417 (1996)

3. Rueckert, D., Sonoda, L.I., Hayes, C., Hill, D., Leach, M., Hawkes, D.: Nonrigid registration using free-form deformations: Application to breast MR images. TMI 18, $712-721(1999)$ 
4. Thirion, J.P.: Image matching as a diffusion process: an analogy with maxwell's demons. MedIA 2, 243-260 (1998)

5. Shen, D., Davatzikos, C.: HAMMER: Hierarchical attribute matching mechanism for elastic registration. TMI 21, 1421-1439 (2002)

6. Tu, Z., Narr, K., Dollár, P., Dinov, I., Thompson, P., Toga, A.: Brain anatomical structure segmentation by hybrid discriminative/generative models. TMI 27, 495508 (2007)

7. Dirk, L., Slagmolen, P., Maes, F., Vandermeulen, D., Suetens, P.: Nonrigid image registration using conditional mutual information. In: Karssemeijer, N., Lelieveldt, B. (eds.) IPMI 2007. LNCS, vol. 4584, pp. 725-737. Springer, Heidelberg (2007)

8. Ojala, T., Pietikainen, M., Maenpaa, T.: Multiresolution gray-scale and rotation invariant texture classification with local binary patterns. PAMI 24, 971-987 (2002)

9. Yershova, A., LaValle, S.: Deterministic sampling methods for spheres and so(3). In: ICRA, pp. 3974-3980 (2004)

10. Wu, G., Qi, F., Shen, D.: Learning-based deformable registration of $\mathrm{mr}$ brain images. TMI 25, 1145-1157 (2006)

11. Glocker, B., Komodakis, N., Paragios, N., Tziritas, G., Navab, N.: Inter and intramodal deformable registration: Continuous deformations meet efficient optimal linear programming. In: IPMI, pp. 408-420 (2007)

12. Tang, T.W.H., Chung, A.C.S.: Non-rigid image registration using graph-cuts. In: Ayache, N., Ourselin, S., Maeder, A. (eds.) MICCAI 2007, Part I. LNCS, vol. 4791, pp. 916-924. Springer, Heidelberg (2007)

13. Yuri, B., Olga, V., Ramin, Z.: Fast approximate energy minimization via graph cuts. PAMI 23, 1222-1239 (2001)

14. Crum, W.R., Rueckert, D., Jenkinson, M., Kennedy, D., Smith, S.M.: A framework for detailed objective comparison of non-rigid registration algorithms in neuroimaging. In: Barillot, C., Haynor, D.R., Hellier, P. (eds.) MICCAI 2004. LNCS, vol. 3216, pp. 679-686. Springer, Heidelberg (2004) 